\title{
SISTEMA DISTRIBUIDO PARA MONITORIZACIÓN Y CONTROL INTEGRADO DE EDIFICIOS
}

\author{
(DISTRIBUTED SYSTEM FOR INTEGRATED BUILDINGS AND MONITORING)
}

\author{
Luis Gomes y A. Steiger-Garção \\ Universidad Nova de Lisboa. Facultad de Ciencias y Tecnología. Dpto. de Ingeniería Electrotécnica. UNINOVA
}

Fecha de recepción: 21-XII-98

PORTUGAL

\section{RESUMEN}

Los trabajos que se presentan en este artículo se relacionan con la aplicación de la tecnología de información en el concepto de edificio inteligente. Será propuesto un sistema distribuido destinado a la monitorización y control integrado del edificio. El sistema puede también servir de apoyo para actividades económicas y servicios. El concepto de domot se presenta (domot = "domus" + "robot") como el tercer inquilino activo del edificio, coexistiendo con los usuarios y administradores siendo capaz de manejar recursos estando a disposición del otro usuario.

El sistema se basa en la definición de dos tipos de modelo de estructura, que se utilizarán como referencia para las partes de hardware y software del sistema. El modelo de referencia de la parte software se basa en una red distribuida heterogénea de puestos de trabajo, ejecutando un conjunto de procesos en que la comunicación entre ellos es basada en el paradigma del cliente-servidor. El modelo de referencia de la parte hardware se basa en una estructura con dos niveles de redes; el primero se utiliza para la comunicación entre los puestos de trabajo de alto nivel (destinados a la supervisión y monitorización) y el segundo estará destinado a garantizar la comunicación entre los controladores de bajo nivel. Estos dos tipos de modelos constituyen la base para una implementación robusta y abierta de una infraestructura que pueda ser utilizada para las actividades económicas y también para los deseos de usuarios individuales, permitiendo progresos sinérgicos.

La interfaz de usuario, que se puede utilizar en cualquier lugar del mundo en que esté disponible una conexión $T C P-I P$, está estructurada jerárquicamente; se proporcionan información y diálogos especificos para la monitorización y control de los diversos tipos de dispositivos y gestión de los mismos y sistemas que estén instalados en el edificio. Los sistemas ya integrados son: detección de intrusos, CCTV y $P A B X$. El control de acceso, aire acondicionado y calefacción, iluminación se añadirán en un futuro próximo.

\section{SUMMARY}

The works to be presented in this paper are related with information technology application on the intelligent building concep. A distributed system targeted for integrated building monitoring and control will be presented. The system is also able to support business activities and services. The concept of domot introduced (domot = "domus" + "robot") as the third active occupant of the building, coexistint with users and managers, and able to manage resources and be at other occupant disposal.

The system relies on the definition of two types of template architectures, to be used as references by the software and hardware parts of the system. The software reference template is based on a heterogeneous distributed network of workstations, running a set of process with interprocess communication based on the client-server paradigm. The hardware reference template is based on a architecture based on two levels of networks; the first one is used for the communication between high-level control workstations (targeted for supervision and monitoring) and the second one is targeted to support low-level controllers communcation. These two types of templates constitute the basis for a robust and open system based implementation of an infrastructure that is able to support both business activities and the single occupant wishes, enabling synergistic developments.

The user interface, which may run anywhere in the world as far as a TCP-IP connection is available, is hierarchically organised; specific information and dialogues are provided for the monitoring and management of different types of devices and systems installed in the building. The systems already integrated are intrusion, CCTV and PABX. Access control and $H V A C$ will follow in the near future- 


\section{Introducción}

El concepto de los edificios inteligentes se ha redefinido varias veces en los últimos años [IBI, 87] [DEGW et al., 95]. Es un área de aplicación multidisciplinaria, a la cual podrían hacer contribuciones expertos de diferentes campos, procediendo de forma complementaria para el beneficio de los ocupantes del edificio y, también, para bien de los propietarios y administradores del edificio.

En este marco de referencia, un edificio inteligente es un ambiente estructurado, equipado con varios sistemas sensoriales y de control y destinado a actividades de monitorización y control (dentro o fuera del edificio), empleando infraestructuras de red heterogéneas que llegan a hacer posible la comunicación de los sistemas informáticos con el ser humano.

Una meta de los actuales trabajos es el desarrollo y la explotación de tal infraestructura, permitiendo la integración de servicios y de actividades. Esto significa que, desde el punto de vista de este trabajo, sólo se resaltarán los aspectos relacionados con las tecnologías de información.

Consideramos que las actividades dentro de los edificios podrían ser ordenadas, optimizadas, controladas más eficientemente y menos costosamente, si un conjunto de infraestructuras complementarias está disponible, actuando de una manera cooperativa y permitiendo la puesta en práctica de nuevas funciones específicas. Estas nuevas funciones se obtienen con una interacción sinérgica de los subsistemas, para obtener una ayuda mejorada de la actividad.

Consideremos un ejemplo relativo a la búsqueda y localización de personas dentro de un edificio (para el desvío de la llamada telefónica, por ejemplo); en este caso, se podían utilizar transmisores personales para determinar la posición de algún ocupante del edificio, mediante el uso de un costoso sistema, basado en la detección de diferentes características de infrarrojos o radiofrecuencias de los transmisores. Una solución alternativa podría utilizar la información proporcionada por un sistema de control de acceso ya instalado y su uso directo por el PABX, con el objeto de obtener un seguimiento transparente para cualquier desvío de la llamada.

Esto significa que, de forma general, la adición de sensores, actuadores y controles electrónicos, con la única misión de lograr el control óptimo de alguna característica de un proceso, no resulta rentable; es necesario integrar sistemas en el edificio para conseguir mejoras globales, como una gerencia mejor y nuevas funcionalidades y servicios.

En este artículo se presentará un sistema destinado a la monitorización y control integrado de edificios inteligentes. Las descripciones anteriores del sistema referido pueden ser encontradas en [Gomes \& SteigerGarção, 94] [Gomes \& Steiger-Garção, 96] [Gomes et al., 97)] [Gomes, 97] [Gomes et al., 98]. A lo largo de todo el desarrollo del sistema descrito, se han utilizado varias técnicas, de sobra conocidas, aplicables para el desarrollo de sistemas que abordan las partes de hardware $(\mathrm{HW})$ y software (SW). Sin embargo, se aprecia la carencia de un entorno integrado que permita la verificación de propiedades o la validación (con la simulación) del sistema distribuido entero.

El problema es demasiado complejo y se necesita de un formalismo adecuado que permita modelizar las diversas partes del sistema así como su integración, comenzando en el nivel del interfaz de usuario y alcanzando el control de bajo nivel asociado a los sistemas empotrados y a los controladores programables instalados en la planta. El uso de los formalismos de los sistemas a eventos discretos, como las redes de Petri (RdP), es muy adecuado para ese propósito, según lo propuesto en [Gomes, 97] [Gomes, 98] [Gomes et al., 98]. Un modelo RdP coloreado no autónomo, nombrado por RdP Reactivas e Jerárquicas, se está utilizando para ese propósito.

\section{Descripción del sistema}

Consideramos que las actividades de desarrollo relativas al concepto de edificio inteligente están ordenadas en tres grupos principales, a saber: desarrollo, configuración y ejecución, véase la figura 1. Esas actividades se asocian a tres grupos completamente distintos de personas y entornos de ordenadores.

El primer grupo considerado, situado en el nivel superior, está asociado al entorno de desarrollo. La meta de esta etapa es el diseño de los diversos sistemas presentes en el edificio, desde problemas de arquitectura y construcción, hasta los sistemas electrónicos.

Aquí, están implicados distintos equipos del proyecto. Desde el punto de vista del grupo que se asocia a la parte "inteligente" del proyecto, meta principal es integrar la información proporcionada por las diversas aplicaciones, en el modelo del edificio. Tienen que considerar entradas de información delCAD arquitectónico, del CAD eléctrico y electrónico y de otras herramientas de $\mathrm{CAD}$, para presentar el edificio en todas sus facetas interesantes. Es peligroso considerar esas actividades aparte de los otros grupos de proyecto, así este grupo "inteligente" es constituido por las personas que, de forma autónoma o integradas en otros equipos del proyecto, aseguran la integración del concepto de edificio inteligente y la cooperación entre los diversos sistemas que se instalarán.

Entre el entorno de desarrollo (nivēl, planos y diseño) y el control de la planta al nivel de la ejecución (nivel inferior, ejecución), consideramos el entorno de configuración. El 


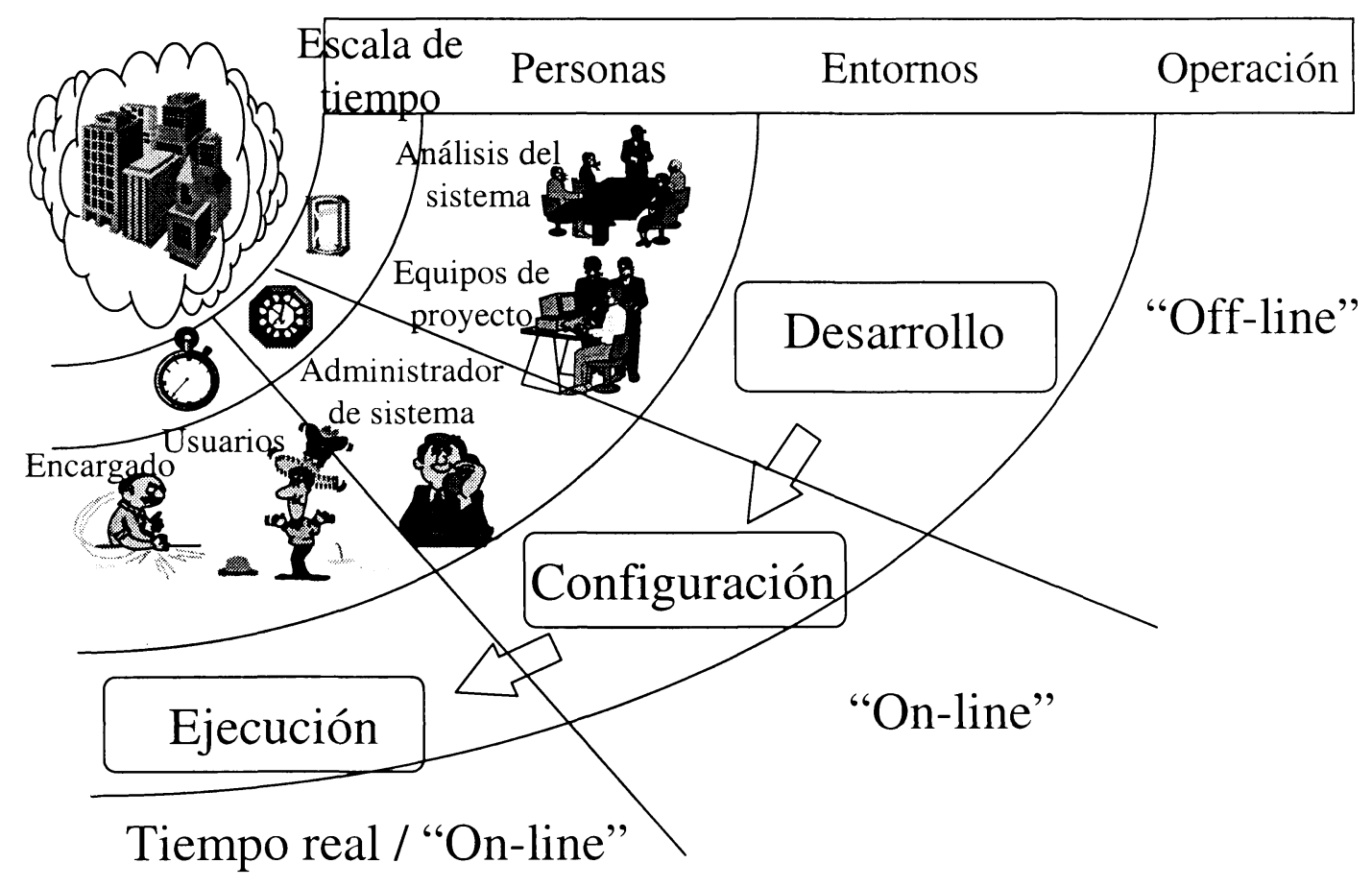

Figura 1.- Actividades de desarrollo relativas al concepto de edificio inteligente.

papel de este entorno es trasladar a los sistemas de planta la solución diseñada.

Aunque se pretenda la puesta en práctica incremental como una meta importante (que permite la adición de nuevos subsistemas uno por uno, a lo largo del tiempo), el entorno de configuración es responsable de: I) registro de las versiones instaladas, II) asegurar la actualización, fácil y "suave", entre las versiones (desde el punto de vista del usuario del edificio), y III) asegurar la instalación fácil de nuevos sistemas, funciones y actividades.

Para hacer el concepto de edificio inteligente de interés para un número más grande de industrias, es necesario tener disponibles algunos modelos de referencia, para permitir desarrollos independientes. Esto significa que se puede disponer de productos procedentes de diversos fabricantes y, en cuanto satisfagan dichos modelos, su integración en la planta del edificio se realizará sin problemas.

Para asegurar la coherencia de estas actividades de integración, proponemos el uso de dos tipos de modelos de referencia, con protocolos abiertos asociados.

El primer modelo de referencia es la arquitectura física (hardware), que se asocia a la definición de un modelo para la interconexión de los sistemas y de los dispositivos (en el nivel físico).

El segundo es el modelo de referencia de la arquitectura lógica (software) y se relaciona con la interconexión de

(c) Consejo Superior de Investigaciones Científicas

Licencia Creative Commons 3.0 España (by-nc) los procesos y aplicaciones en el sistema distribuido. Como complemento a este modelo de referencia, es preciso conocer los protocolos específicos que serán utilizados por cada uno de los procesos; los protocolos abiertos y los mensajes textuales deberían utilizarse siempre que sea posible y adecuado.

Más allá de los modelos de referencia, proponemos que dos características más sean consideradas cruciales para lograr alcanzar el éxito. Por una parte, debemos utilizar estándares $\mathrm{y}$, por la otra, debemos adoptar una actitud equilibrada.

El uso de estándares, como STEP de la ISO para el intercambio de datos del producto, permitirá el intercambio de datos entre el entorno de desarrollo y el entorno de ejecución, con la reducción al mínimo de los errores y de la mano de obra en el entorno de la configuración.

Una actitud equilibrada indica que la activación de una cierta característica sinérgica específica tiene que ser elegida explícitamente por el usuario y no será utilizada de una manera automática para todos los usuarios; el utilizador tiene que tener la oportunidad de activarla, desactivarla o de configurarla siempre que quiera. Volviéndose al ejemplo del seguimiento de la llamada referido en la sección anterior, debe serposible para el usuario activary desactivar tal característica.

Las subdivisiones siguientes se dedican- a presentar individualmente los aspectos de la arquitectura física y lógica del sistema de monitorización y control. 


\section{1 Arquitectura fisica}

En situaciones comunes, los sistemas instalados en la planta del edificio son heterogéneos. Para asegurar su integración dentro de un entorno común, es necesario utilizar estándares y protocolos abiertos. Hay una gran cantidad de protocolos en uso, algunos de ellos son propietarios, otros están abiertos y otros se pueden conocer previo pago de licencia. Se admite ampliamente que ningún protocolo puede convertirse en "el único", en un futuro próximo. Sin embargo, hay alineaciones claras: en Europa, el EIB (European Installation Bus), utilizado por la European Installation Bus Association, es el que más utilizan las industrias europeas, mientras que el BACnet (Building Automation and Control Network), utilizado por el ASHRAE -American Society of Heating, Refrigeration and Air Conditioning Engineers- parece ser el preferido por la industria americana; para las instituciones académicas y de $\mathrm{I}+\mathrm{D}$, la preferida es utilizar la red mundial IP (Internet Protocol), normalmente disponible dentro del campus de la universidad.

Conviene mencionar también algunos otros protocolos, a saber: el CEBus (Consumer Electronics Bus), utilizado por EIA - Electronic Industries Association- de los EE UU; el LonTalk, desarrollado por Echelon, de los EE UU; varios field-buses, como ProfiBus y el WoldFip; el N2-Bus, desarrollado por Johnson Controls, Inc., de los EEUUy elprocoloX-10, para las aplicaciones de "pequeña escala".

En los trabajos relativos a este artículo, se han utilizado extensamente la red IP y el protocolo TCP-IP (Transfer Control Protocol - Internet Protocol), debido a varias razones (obviamente, la primera de ellas es que está disponible, sin costes adicionales, dentro de nuestro campus de la universidad): -es un protocolo estándard, utilizado por todas las plataformas interesantes;

-ayuda implícita para el modelo de computación clienteservidor;

-la gestión de usuarios es transparente en lo que respecta a la ubicación de las estaciones y a los medios físicos que permiten la comunicación entre ellos;

-admite de forma intrínseca la instalación incremental de nuevos sistemas y estaciones.

La figura 2 muestra un sistema distribuido de dos niveles que se ha utilizado como referencia.

El nivel superior consta de varias estaciones de trabajo interconectadas con una red de área local (LAN), por ejemplo una LAN Ethemet basado en TCP-IP. Estas estaciones de trabajo están dedicadas a las tareas de monitorización y de supervisión.

El nivel inferior es compuesto por los sistemas de control dedicados, por ejemplo, sistemas incrustados para el control local, interconectados por una cierta red dedicada, típicamente una red serial como RS-485 o RS-232.

Es una arquitectura extensamente usada como referencia para los sistemas distribuidos (por ejemplo, la mayoría de los field-buses industriales tienen una estructura similar).

Con respecto a la figura 2 , se muestran varios sistemas, a saber: el subsistema de CCTV (televisión en circuito cerrado), el subsistema del PABX (sistema privado de teléfonos), el subsistema de intrusión, el subsistema del control de acceso y el subsistema de la gestión de energía eléctrica. Con respecto a los subsistemas de control de acceso y energía no están instalados en la planta; el resto de los subsistemas presentados están en activo.

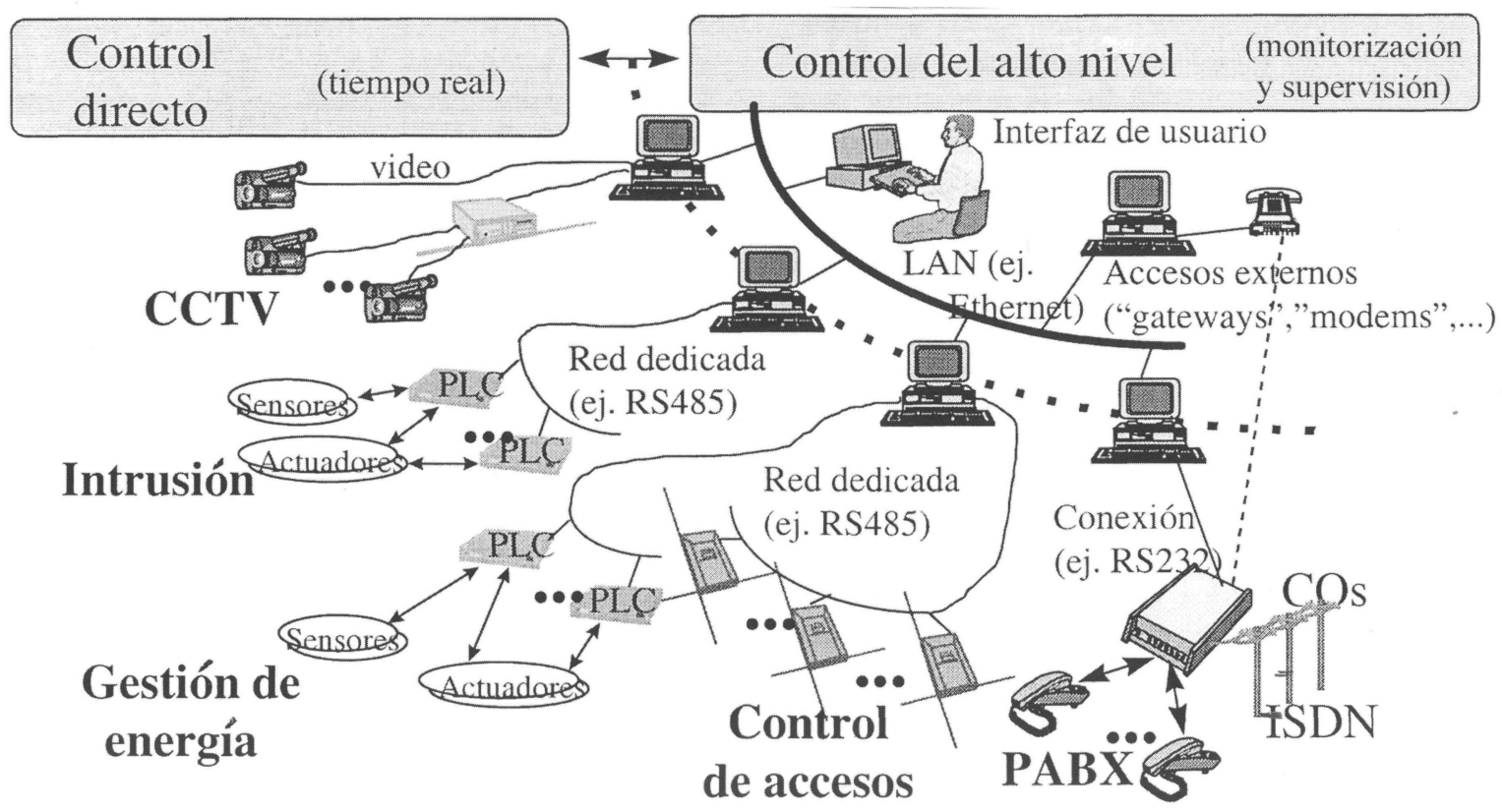

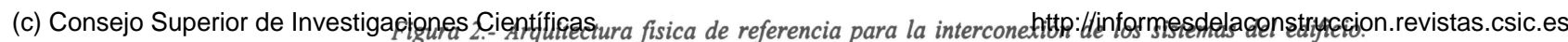
Licencia Creative Commons 3.0 España (by-nc) 
Otro componente muy importante del sistema es el que se denota mediante "accesos externos" (esquina superiorderecha de la figura) y que es responsable por la conexión con el mundo exterior y que garantiza la conectividad a la WWW (World Wide Web). Aunque no sea evidente en la figura, los diversos subsistemas se puden interconectar a través de una misma red común (siempre y cuando los protocolos que se utilizan lo permitan). Además, no es necesario tener una estación que centralice toda la información asociada a un subsistema; esto significa que, por ejemplo, la información asociada al sistema de la intrusión se puede manejar por un conjunto de estaciones de trabajo, cada una de las cuales estará asociada a una parte del subsistema e instalada en un lugar diferente, permitiendo un buen equilibrio de las cargas de trafico IP en las diversas ramificaciones de la red de área local.

\subsection{Arquitectura lógica}

Según lo ya indicado anteriormente, el edificio inteligente es un ambiente heterogéneo, compuesto por diversos subsistemas autónomos y (esperemos) cooperativos. Para alcanzar una explotación completa de tal infraestructura es necesario tener una arquitectura sencilla para el usuario, de modo que permita unos diálogos fáciles y eficientes entre diversos tipos de usuarios y la infraestructura del edificio.

Así pues, es necesario tener un conjunto de entidades de computación responsables de garantizar esos diálogos. Es decir algunas entidades de computación (aplicaciones) que podrían manejar una cierta clase de recursos y pueden estar a la disposición del usuario; a estas entidades se les dará el nombre de domots, que proviene de la agregación de las palabras "domus" y "robot".

El segundo modelo de referencia, que está relacionado con la arquitectura lógica de interconexión de los procesos, esto es desde un punto de vista del sofware, estará caracterizado en términos de diversos tipos de domots.

La configuración se basa en una estructura cliente-servidor utilizando mensajes inter-procesos basados en los socketes TCP-IP(figura 3). Esta actitud permite el uso de estaciones de trabajo heterogéneas, pertenecientes a una gama que va desde las más económicas a las más costosas.

El puente con los procesos de control de bajo nivel, que normalmente están asociados a un hardware dedicado, se logra con el uso de pasarelas y de conversores de protocolo específicos; por ejemplo, se puede considerar al convertidor entre una red TCP-IP y una red RS-485.

En este sentido, el conjunto de procesos se divide en tres grupos:

-grupo de las actividades de edificio, asociado con el control de alto nivel y actividades de supervisión y monitorización, es identificado como domots maestros desde el punto de vista del usuario del edificio; entre los domots maestros ya desarrollados se encuentra una aplicación de interfaz de usuario, que se presentará en la subdivisión siguiente; en términos generales, los domots maestros son los responsables de gestionar recursos específicos, para proveer información relacionada con el análisis de distintas actividades del edificio o para admitir sistemas auxiliares, como sistemas expertos y otros sistemas de apoyo para la toma de decisiones, responsables de la supervisión de recursos específicos o de la emisión de diagnósticos y sugerencias para los usuarios y administradores, de forma autónoma o semiautónoma;

-grupo de captura de datos, que constituye la base del sistema de monitorización en sí y consta de un conjunto de

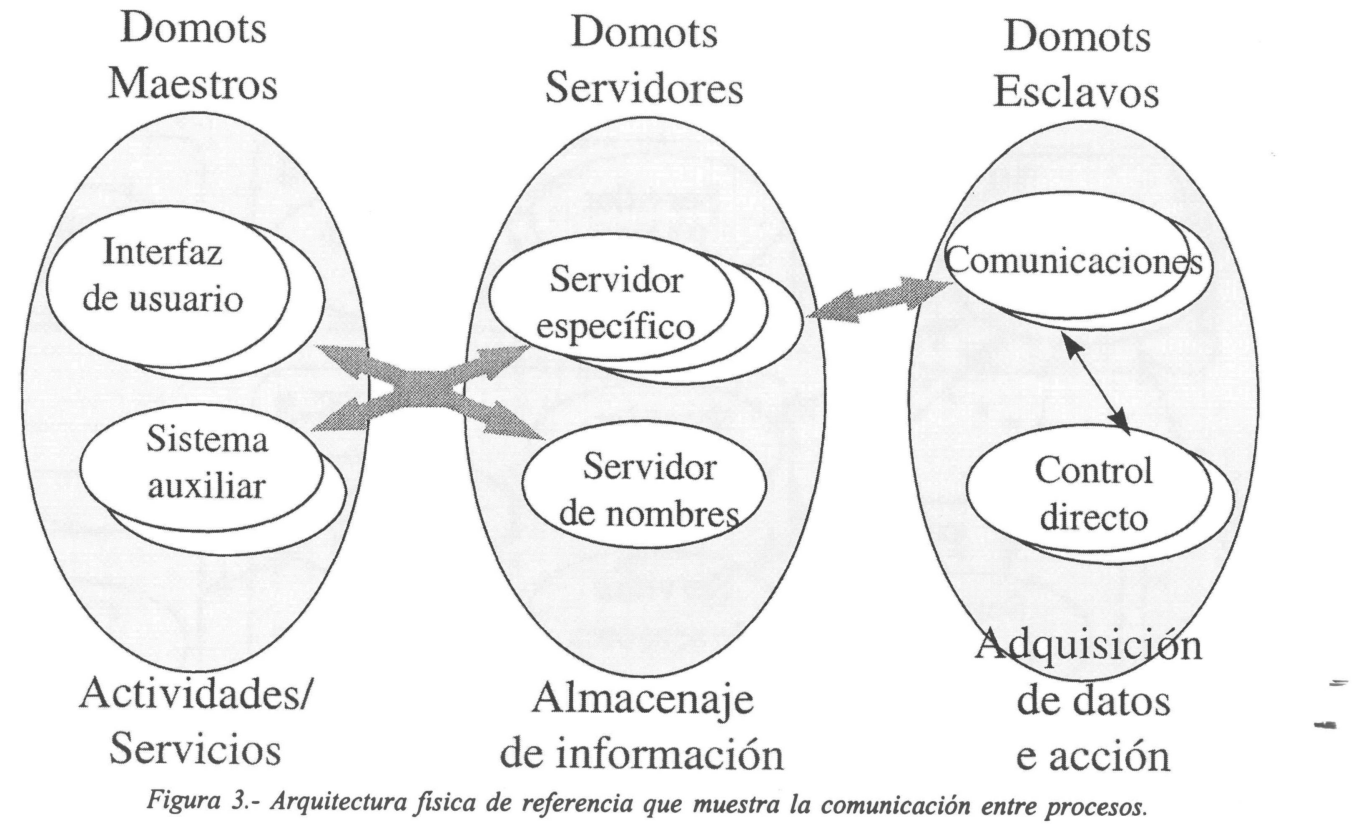

(c) Consejo Superior de Investigaciones Científicas

http://informesdelaconstruccion.revistas.csic.es

Licencia Creative Commons 3.0 España (by-nc) 
servidores asociados a los recursos específicos que se supervisarán; los procesos de este grupo se identifican como domots servidores y son responsables de proporcionar información actualizada sobre el estado de los recursos y de asegurar el almacenamiento a largo plazo de la información asociada;

-grupo de adquisición de datos y de la acción asociada al control de bajo nivel; los procesos en este grupo se identifican como domots esclavos y son responsables de los actuadores de salida y de la adquisición de datos de la planta del edificio; estos procesos pueden ejecutarse en controladores dedicados, sistemas incrustados u otras plataformas de fines generales y se adecúe a ellos a través de servidores de comunicaciones, responsables de la conversión de protocolos (por ejemplo, el puente ya mencionado de la comunicación de TCP-IP a la red RS-485).

Los domots servidores, es decir los servidores de captura de datos, se ordenan en dos grupos:

-El primero agrupa los servidores específicos, que son responsables de recoger información y de manejar un recurso específico; entre los ejemplos se cuentan los servidores que gestionan los detectores de presencia (sistema de intrusión), cámara de vídeo, sistemas de control de acceso, entre otros.

-El segundo agrupa a los servidores auxiliares, a saber: el servidor de nombres; el servidor de nombres es vital para la operación de todos los sistemas; él es responsable de proporcionar información relacionada con todos los componentes del sistema, en forma de una etiqueta (nombre simbólico) y el servidor o servidores específicos en que se puede obtener información (por ejemplo, en el caso de un detector de presencia su actual estado, su localización y demás).

\subsection{Entorno Experimental}

En la actual subdivisión, se presenta una descripción abreviada de una aplicación de interfazde usuario destinada a la monitorización del edificio junto con los procesos domots asociados.

La figura 4 muestra la distribución de procesos en el entorno experimental:

-la aplicación del interfaz de usuario, ejecutándose en una estación denominada la PCU; la figura 5 muestra un volcado de pantalla del uso típico de este domot; dado que está destinado a la monitorización del edificio, se admite la navegación por diferentes pisos y espacios; los iconos gráficos presentados en la planta se pueden utilizar para conseguir la información específica asociada al dispositivo (por ejemplo, la imagen asociada a una cámara fotográfica, o al estado del detector de presencia);

-cuatro estaciones, denominadas PCS-1 a PCS-4, en que se ejecutan cinco domots servidores; el primero es el servidor de nombres ya mencionado, mientras que los cuatro servidores restantes se relacionan con un recurso específico: el servidor de la planta proporciona la información geométrica asociada a todos los espacios del edificio (o, eventual, de un conjunto de edificios) y los otros tres servidores (imágenes, sensores y PABX) son responsables de la interacción con los recursos específicos;

-tres controladores lógicos programables, denominados PLC-1 a PLC-3, responsables de la adquisición de datos de la planta y también de la activación de actuadores, así como una centralita telefónica.

La estructura de la aplicación domot permite un ajuste dinámico (durante la ejecución) al entorno que se quiera

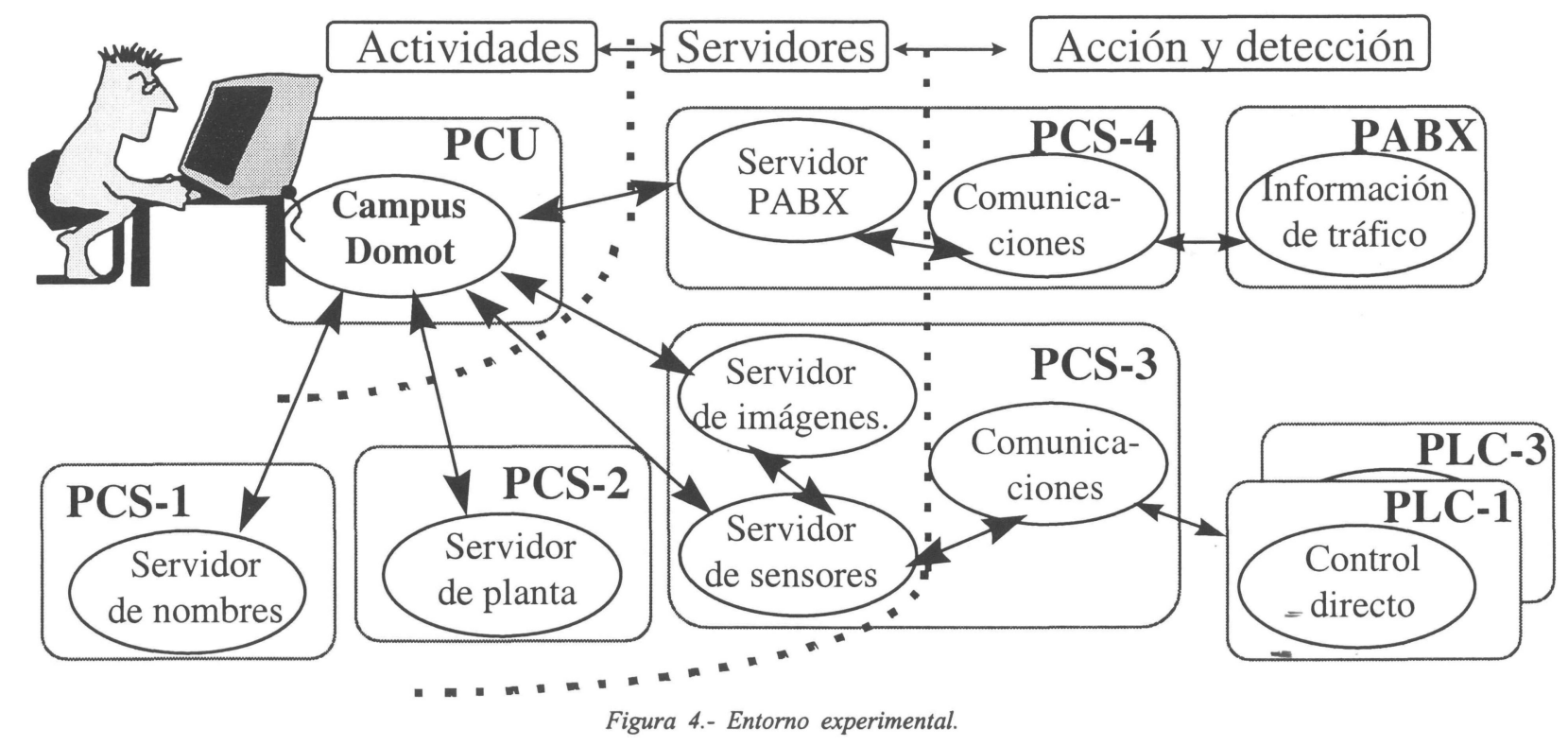




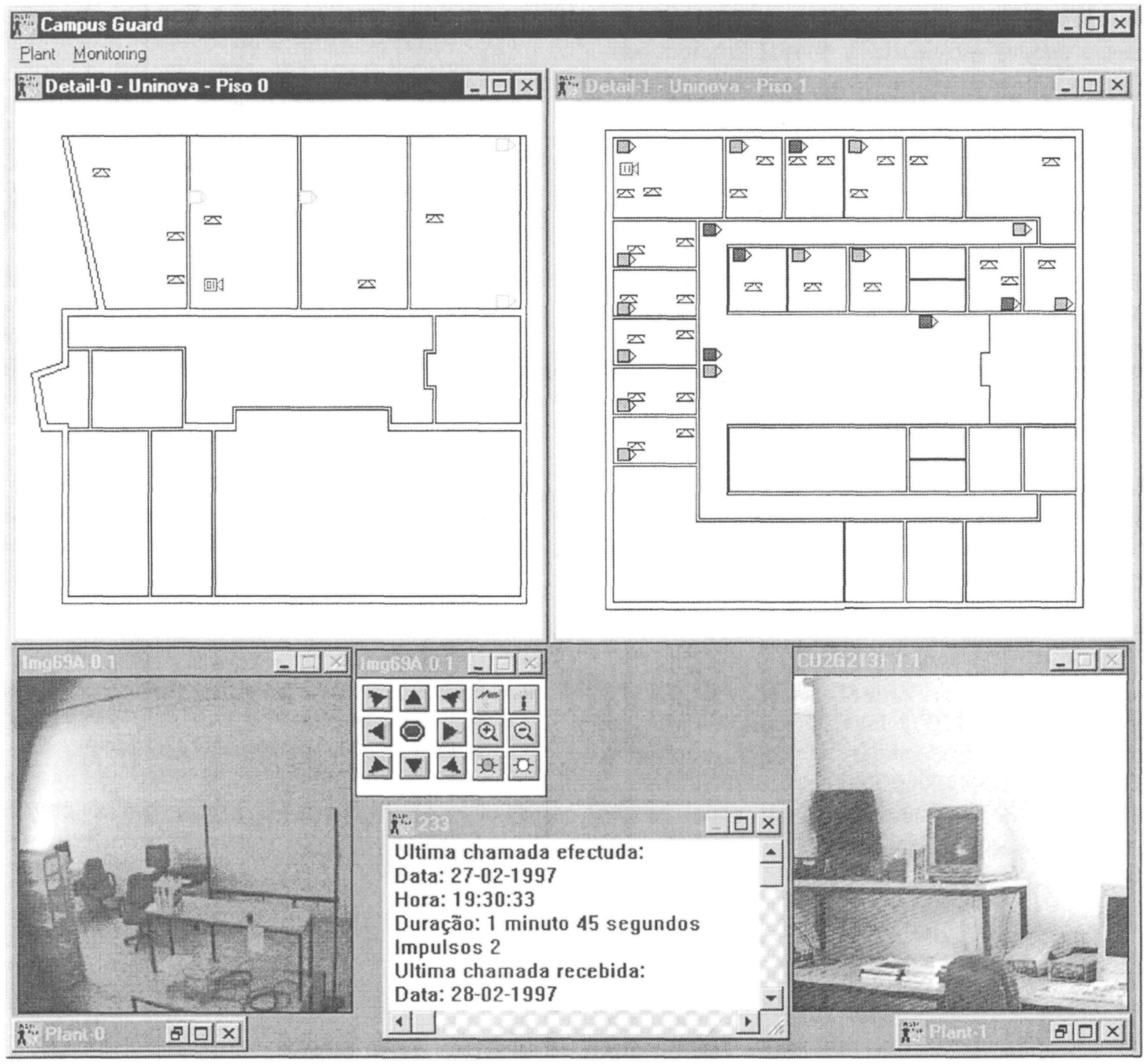

Figura 5.- Interfaz de usuario para monitorizar el edificio.

monitorizar. Esto significa que toda la información que se presenta se obtiene durante la ejecución, a partir de los nombres y de las características geométricas de los espacios, incluyendo el estado actualizado de los distintos dispositivos. Esto se logra por acceso al servidor de nombres al principio de la ejecución del proceso, que dará la información sobre la localización de los otros servidores; de los otros servidores, uno puede conseguir la información sobre todos los dispositivos instalados en la planta. En este sentido, la localización del puerto de comunicaciones del domot servidor de nombres (dirección $I P$ y puerto) es lo único que tiene que saber el domot de interfaz de usuario para ser capaz de monitorizar el edificio (o grupo de edificios).

Debido a las características del sistema, estas actividades de monitorización pueden producirse en cualquier lugar de la red IP, lo cual quiere decir que en cualquier lugar del mundo, siempre y cuando los retrasos de las comunicaciones sean aceptables. Esta línea de experimentos se ha venido a analizar dentro del proyecto CYTED SInEd-Integración de Sistemas y Actividades en Edificios Inteligentes (www.uninova.pt/sinted).

\section{Conclusiones}

En conclusión, se puede decir que los modelos de referencia propuestos -que son aplicables a la interconexión de la arquitectura física (hardware) y de la arquitectura lógica (software)- parecen ser adecuados para la integración y gestión del uso sinérgico de un conjunto de sistemas heterogéneos presentes en edificios inteligentes. El prototipo que se utiliza en UNINOVA integra los subsistemas de intrusión, de CCTV y de PABX y está destinado actualmente al soporte de actividades de monitorización.

Los modelos propuestos de referencia permiten una sencilla integración incremental de nuevos subsistemas que es el objetivo perseguido. Dos ejemplos son el control

http://informesdelaconstruccion.revistas.csic.es 
de acceso y los sistemas de ahorro de energía eléctrica; esto permitirá nuevas actividades y nuevas funcionalidades, produciendo un significativo impacto sobre la forma en que los ocupantes del edificio hacen uso de su infraestructura.

\section{BIBLIOGRAFÍA}

(1) [DEGW et al., 95] DEGW (London) and TEKNIBANC (Milan); 1995; The Intelligent Building in Europe-Executive summary; New methods and technologies in planing and construction of Intelligent Buildings, Proccedings of IB/IC Intelligent Building Congress'95 Tel-Aviv, Israel; Editor A. Lustig;pp. 186-209.

(2) [GOMES \& STEIGER-GARÇÃO, 94] LUIS GOMES; A. STEIGER-GARÇÃO; 1994. "Towards different buildings..."; Conferencia Internacional sobre el control Automático en el Ahorro de Energía; La Habana, Cuba; febrero, 21-25.

(3) [GOMES \& STEIGER-GARÇÃO, 96] LUIS GOMES; A. STEIGER-GARÇÃO; 1996. "Domots are coming ¿Or How to manage building automation in a balanced way?"; BASYS '96 - 2nd IEEE/ECLA/IFIP International Conference on Architectures and Design Methods for Balanced Automation; Lisbon, Portugal; 17-19 june 96; in Balanced Automation Systems II; Luis M. Camarinha-Matos and Hamideh Afsarmenesh(Eds), pp. 55-64; Chapman \& Hall; ISBN0-41278890-X.
(4) [GOMES et al., 98] LUIS GOMES; ANIKÓ COSTA; CARLOS SOARES; JOAO-PAULO BARROS; ADOLFO STEIGER-GARÇÃO; 1997. "Campus-Guard: a domot targeted for integrated building monitoring and control"; 2 nd Int. Conference on Intelligent Buildings; Tel-Aviv, Israel; 4-6 marcha 1997; in New Methods and Technologies in Planning and Construction of Intelligent Buildings II; pp. 53-64; IB/IC Intelligent Buildings Congress, Israel, ISSN 0793-2448.

(5) [GOMES elal.,98]LUIS GOMES, JOAO PAULOBARROS, ANIKÓ COSTA; 19-21 october 1998; "Towards Integrated Modelling of Intelligent Builging Systems"; Second European Conference on Product and Process Modelling in the Building Industry; R. Amor (Ed.); Clowes Group; ISBN 186081249 $\mathrm{X}$.

(6) [GOMES, 97] LUIS GOMES; 1997; "Redes de Petri Reactivas e Hierárquicas - integraçăo de formalismo no projeto de sistemas reactivos de tempo-real"; PhD Thesis; Universidade Nova de Lisboa, Faculdade de Ciências e Tecnologia; 318 págs.

(7) [GOMES, 98] LUIS GOMES; 1998; "Redes de Petri Reactivas e Hierárquicas: características e aplicações em edificios inteligentes"; II Workshop SInEd sobre Edificios Inteligentes; Varadero, Cuba febrero 1998.

(8) [IBI, 87]IBI-Intelligent Buildings Institute; 1987; Intelligent Building Definition - Guideline; Public report; Washington D.C. EE UU.

\section{Publicación del Instituto Eduardo Torroja - CSIC}

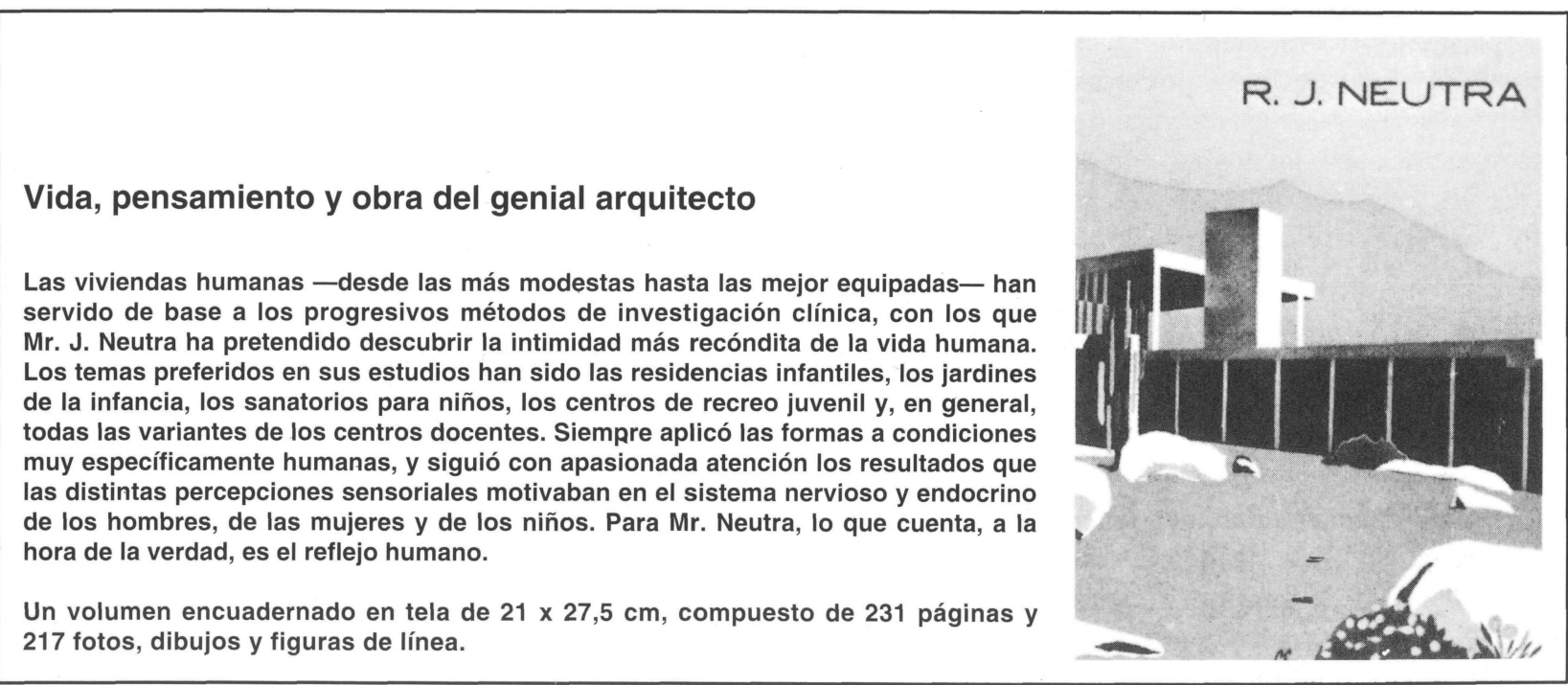

